\title{
ACTIVE CONTROL OF HYPERSONIC SHOCK LAYER DISTURBANCES
}

\author{
T. V. Poplavskaya ${ }^{1}$ and A. A. Maslov ${ }^{2}$ \\ ${ }^{1}$ Institute of Theoretical and Applied Mechanics \\ Siberian Branch of the Russian Academy of Sciences \\ Novosibirsk, Russia \\ ${ }^{2}$ Novosibirsk State University \\ Novosibirsk, Russia
}

This is a brief review of the investigations of receptivity and control of hypersonic shock layers. The present paper describes comprehensive numerical and experimental investigations of evolution of disturbances generated in the hypersonic viscous shock layer (VSL) on a flat plate by external acoustic waves and by perturbations introduced into the shock layer from the surface of model. The active control of intensity of pulsations is possible because both external acoustic waves and the periodic controlled disturbances introduced on the plate surface generate, in a shock layer, entropy-vorticity disturbances with identical spatial distributions and phase velocities.

\section{INTRODUCTION}

The receptivity of a hypersonic shock layer to external and internal disturbances plays an important role in formation of a spectrum of initial disturbances and influences laminar-turbulent transition in a hypersonic boundary layer. In this connection, the investigation of wave processes in a hypersonic shock layer and development of methods of controlling their intensity is an important scientific problem. In high-velocity high-altitude flight, the entire space between the surface of the flying vehicle and the bow shock wave (SW) even at a large distance from the leading edges is the viscous flow zone where the so-called viscous shock layer is formed. The VSL consists of a thick boundary layer and a thin layer of an inviscid flow behind the SW. Like the boundary layer, the laminar shock layer is unstable, and perturbations developed in this layer induce its transition to the turbulent flow regime. The evolution of perturbations in supersonic flows with Mach numbers 6 and 10 was studied by Egorov et al. [1, 2] and Ma and Zhong $[3,4]$. However, mechanisms governing emergence and development

This is an Open Access article distributed under the terms of the Creative Commons Attribution-Noncommercial License 3.0, which permits unrestricted use, distribution, and reproduction in any noncommercial medium, provided the original work is properly cited. 
of perturbations in the VSL with higher Mach numbers may differ from those investigations with lower Mach numbers.

The present paper describes the results of direct numerical simulations (DNS) of receptivity and evolution of perturbations in a VSL on a flat plate for a very high Mach number $\left(\mathrm{M}_{\infty}=21\right)$ and a moderate Reynolds number $\left(\operatorname{Re}_{L}\right.$ $=1.44 \cdot 10^{5}$ ). The problem of interaction of the shock layer with free-stream acoustic disturbances of slow and fast modes was studied by solving twodimensional (2D) Navier-Stokes equations. The computed results are compared with characteristics of density fluctuations measured in experiments with the same flow parameters in hypersonic nitrogen wind tunnel at the Khristianovich Institute of Theoretical and Applied Mechanics (Siberian Branch of the Russian Academy of Sciences (ITAM).

\section{NUMERICAL FORMULATION OF THE PROBLEM}

Direct numerical simulations involved solving 2D Navier-Stokes equations written as a system of conservation laws. The system is closed by the equation of state for a perfect gas. Two-dimensional Navier-Stokes equations are solved by high-order shock-capturing schemes [5]. Convective terms of the NavierStokes equations are approximated by the 5th order monotonicity-preserving scheme [6]. The diffuse terms in system are approximated by the 4 th order finite differences [7]. Integration in time is performed by the 3rd order Runge-Kutta method [8]. Details of the numerical method can be found in $[9,10]$.

The computational domain is a rectangle. A part of the lower side coincides with the plate surface. The left inflow boundary is located at a distance of a few computational cells upstream from its leading edge. The height of the computational domain is chosen such that the bow SW emanating from the leading edge does not interact with the upper boundary. The right outflow boundary is moved downstream from the trailing edge so that the flow in the exit cross section is fully supersonic.

The steady flow is computed first with a uniform hypersonic flow set on the inflow and top boundaries. On the outflow boundary, the solution is extrapolated from inside the computational domain. As the rarefaction effects in the problem considered are fairly significant, the boundary conditions on the plate take into account the velocity slip and temperature jump. A uniform computational grid consisted of 1050 cells in the streamwise direction and 240 in the crossflow direction. The computations involved operation of up to 10 processors in the Siberian Supercomputer Center.

Then, the problem of interaction of a VSL with external acoustic disturbances propagating in the streamwise direction was solved. The variables on the left boundary were set as a superposition of the steady main flow and a planar monochromatic acoustic wave: 


$$
\left(\begin{array}{c}
u^{\prime} \\
v^{\prime} \\
p^{\prime} \\
\rho^{\prime}
\end{array}\right)=A\left(\begin{array}{c} 
\pm \cos \theta \\
\mp \sin \theta \\
1 \\
1
\end{array}\right) \exp \left[i\left(k_{x} x+k_{y} y-\omega t\right)\right]
$$

Here, $u^{\prime}, v^{\prime}, p^{\prime}$, and $\rho^{\prime}$ are the fluctuations of the longitudinal and transverse velocity, pressure, and density, respectively; $\theta$ is the angle of propagation of the external acoustic wave; $A$ is the disturbance amplitude; $t$ is the time; $k_{x}=k \cos \theta$ and $k_{y}=-k \sin \theta$ are the components of the wave vector related to the frequency $\omega=2 \pi f L / c_{\infty}$ by the dispersion expression $k=\omega /\left(\mathrm{M}_{\infty} \cos \theta \pm 1\right)$. In the abovegiven relations, the upper (lower) index corresponds to the fast (slow) acoustic wave. In dimensionless relations, the density and temperature disturbances are normalized to their free-stream values, the velocity perturbations are normalized to the velocity of sound in the free stream $c_{\infty}$, and the pressure disturbances are normalized to $\rho_{\infty} c_{\infty}^{2}$.

In the experiment, artificial disturbances in the form of periodic injection and suction were locally introduced from the leading edge of the plate. In the numerical solution of the problem, they were modeled by imposing the following boundary condition for the transverse flow rate at a certain part of the surface near the leading edge of the plate:

$$
\frac{\left.\rho v^{\prime}\right|_{y=0}}{\rho_{\infty} c_{\infty}}=A \sin \left(\pi \frac{x-x_{1}}{x_{2}-x_{1}}\right) \sin \pi f .
$$

Here, $v^{\prime}$ are the velocity fluctuations in the direction normal to the plate surface; $\rho$ is the local density; $A$ is the amplitude; and $x_{1}$ and $x_{2}$ are the boundaries of the region where the controlled disturbances were introduced. After introduction of disturbances, the Navier-Stokes equations were integrated until the unsteady solution reached a steady periodic regime.

The simulations of density fluctuations turned out to be in good agreement with the measurements of characteristics of density fluctuations in the shock layer $[9,10]$ and with the computations by the locally parallel linear stability theory with allowance for the influence of the SW [11] for the wall temperature of the model $T_{w}=300 \mathrm{~K}$.

\section{EXPERIMENTAL EQUIPMENT AND DIAGNOSTIC METHOD}

The experiments were conducted in a hypersonic nitrogen wind tunnel at ITAM.

The flat plate was made of low-carbon steel. Its planform was a trapezoid with a length of $240 \mathrm{~mm}$, width at the leading edge of $100 \mathrm{~mm}$, and width at the trailing edge of $80 \mathrm{~mm}$. The plate thickness was $8 \mathrm{~mm}$; the sharp leading edge of 
the plate was shaped as a 7 degree wedge with a bluntness radius of $\cong 0.05 \mathrm{~mm}$. The side edges of the plate were also the wedges with an angle of $20^{\circ}$. The surface temperature was equal to $300 \mathrm{~K}$ and monitored by a copper-Constantan thermocouple. The flat plate was mounted at a zero angle of attack to the flow direction.

Perturbations were introduced into the shock layer on the flat plate by a cylindrical oblique-cut gasdynamic whistle [12]. External acoustic waves (natural disturbances) are the inherent noise of the flow in a wind tunnel.

The mean density and density fluctuations were measured by the method of electron-beam fluorescence. This method adapted to measurements of the characteristics of density fluctuations (spectrum and phase of fluctuations, phase velocity of disturbances) was described in detail in [13].

\section{RESULTS}

Figure 1 demonstrates the instantaneous fields of density fluctuations in the shock layer for a slow external acoustic wave, which were obtained in computations and experiments. The spatial patterns of the fields of fluctuations are in good agreement. The most intense density fluctuations are observed on the SW and on the edge of the viscous boundary layer.

The main specific feature in the formation of the field of density fluctuations is the mechanism of generation of entropy-vortex disturbances and their propagation inside the shock layer. Instant vector field of velocity pulsations is shown in Fig. 2. Here, you can see pairs of vortexes with opposite rotating. This result is also confirmed by the linear theory of interaction of plane waves with the SW [14]. Figure 3 shows the regions of existence of perturbations of different modes in the shock layer which were calculated in accordance with theory [14]. As was shown by measurements and computations, the SW inclination angle $\varphi$ in the case considered changed from $16^{\circ}$ near the leading edge of the plate to $7.5^{\circ}$ at the end of the plate. The discriminating curves 1 and 2 indicate the critical angles of propagation of slow (under curve 1) and fast (above curve 2) acoustic waves behind SW with $\mathrm{M}_{\infty}=21$ depending on the SW inclination angle. According to the linear theory of interaction of perturbations with the SW, the entropy-vortex disturbances propagate inside the region bounded by curves 1 and 2 , while the acoustic waves decay exponentially.

For verification of computations, the amplitudes of density fluctuations on the SW were measured by the method of electron-beam fluorescence of nitrogen [13]. Figure 4 demonstrates the comparison of experimental and numerical data for slow acoustic disturbances for different frequencies on the SW (Fig. 4a) and on the upper boundary layer edge (Fig. 4b). The computed and experimental curves are in good agreement. 


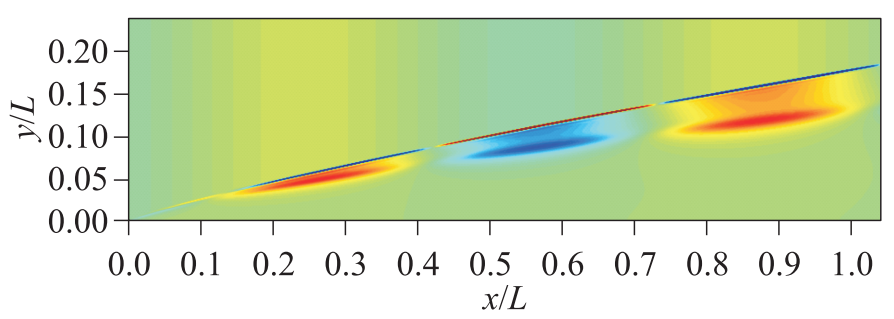

(a)

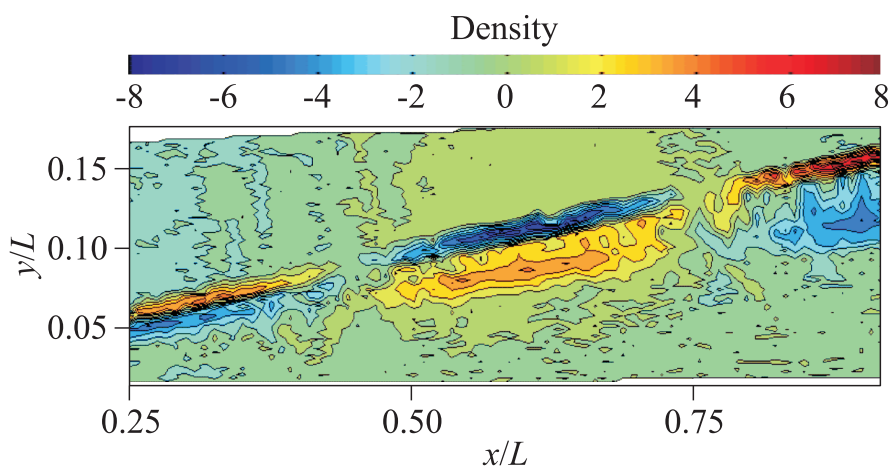

(b)

Figure 1 The instantaneous fields of density fluctuations in the shock layer for a slow external acoustic wave, which were obtained in computations $(a)$ and experiments $(b)$ : $\mathrm{M}_{\infty}=21, \operatorname{Re}_{L}=1.44 \cdot 10^{5}, f=9 \mathrm{kHz}, A=0.03$, and $\theta=0^{\circ}$. (Refer Poplavskaya and Maslov, p. 209.)

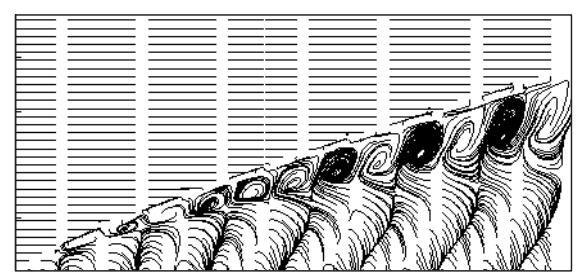

Figure 2 Vector field of velocity pulsation: $\mathrm{M}_{\infty}=21, \mathrm{Re}_{L}=1.44 \cdot 10^{5}, f=9 \mathrm{kHz}$, $A=0.03$, and $\theta=0^{\circ}$

For profound understanding of the mechanism of receptivity of the shock layer, it is also necessary to study the case of localized receptivity. Figure 5 demonstrates the isolines of density fluctuations obtained in computations and experiments. They resemble the flowfield generated by external acoustic disturbances. It is seen that the field of intense fluctuations occupies the region between 


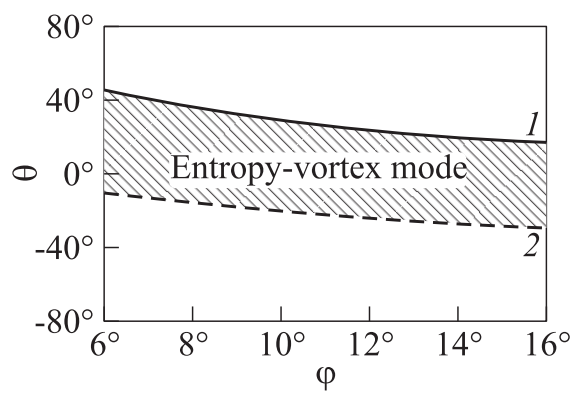

Figure 3 The critical angles of propagation of slow acoustic waves in an undisturbed hypersonic flow with $\mathrm{M}_{\infty}=21$ vs. the angle of inclination of the shock wave

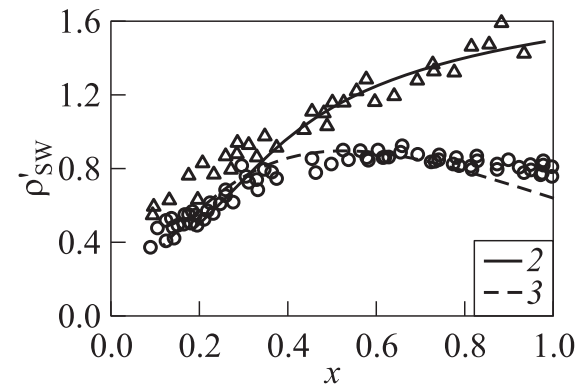

(a)

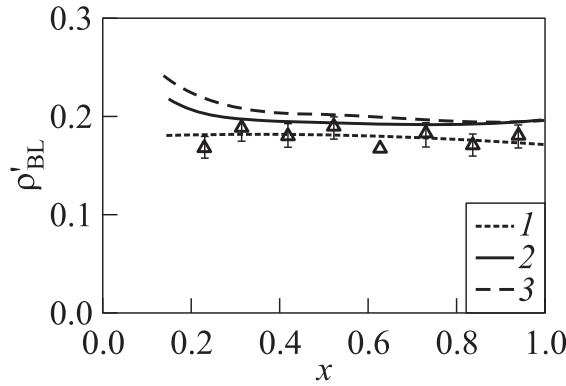

(b)

Figure 4 Comparison of experimental (symbols) and numerical (curves) data for slow acoustic waves for different frequencies on the SW $(a)$ and on the upper boundary layer edge $(b)(1-9 \mathrm{kHz} ; 2-19.2$; and $3-38.4 \mathrm{kHz}) ; \mathrm{M}_{\infty}=21, \operatorname{Re}_{L}=1.44 \cdot 10^{5}$, $A=0.03$, and $\theta=0^{\circ}$

the SW and the boundary layer edge, which corresponds to the maximum gradient of the mean density, as in the case of external acoustic perturbations. Again, there are two maximums of intensity of density fluctuations; one of them (higher peak) is located on the SW, the second maximum (lower peak) is located on the boundary layer.

The computed and experimental density fluctuations in the shock layer for the case of periodic perturbations of the blowing-suction type are compared in Fig. 6. Symbols show experimental amplitudes obtained by the electron beam fluorescence method. The computed and experimental data are in good agreement on the SW and on the upper edge of the boundary layer.

Similar flowfields provide a possibility of active control of the evolution of disturbances in the shock layer. The blowing-suction of the corresponding am- 

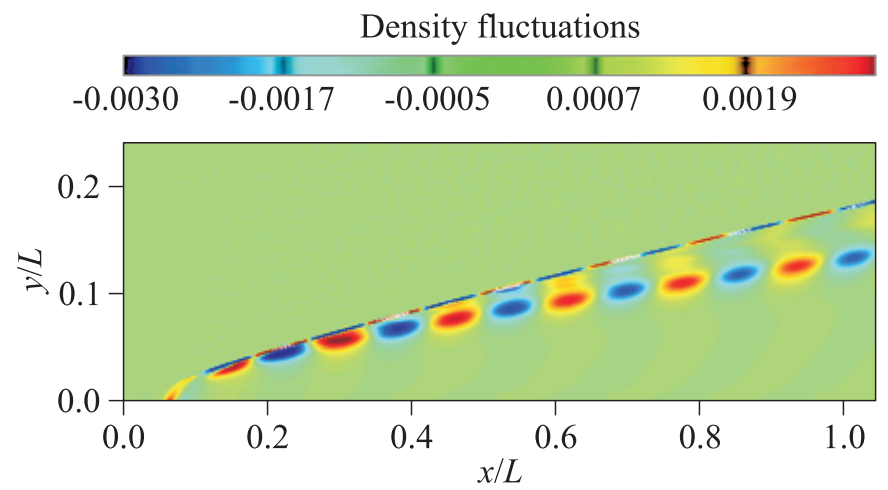

(a)

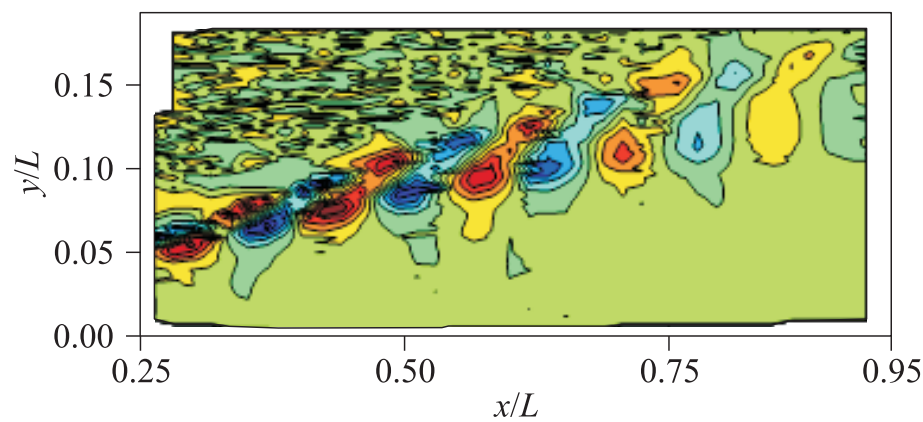

(b)

Figure 5 The instantaneous fields of density fluctuations in the shock layer induced by periodic perturbations from plate surface at a frequency $f=40 \mathrm{kHz}$, which were obtained in computations $(a)$ and experiments $(b): \mathrm{M}_{\infty}=21, \operatorname{Re}_{L}=1.44 \cdot 10^{5}$, $f=40 \mathrm{kHz}$, and $A=0.03$. (Refer Poplavskaya and Maslov, p. 211.)

plitude and phase can be used to delay significantly or to amplify the development of disturbances. The computed results for the fast acoustic waves are shown in Fig. 7. The periodic perturbations of the blowing-suction type from the flat-plate surface were inserted in the opposite phase (Fig. $7 c$ ) or in-phase (Fig. $7 d$ ) to external acoustic disturbances and obtained significant suppression of density fluctuations on the boundary-layer edge.

The principle opportunity of suppression and amplification of disturbances using simultaneous influence on the shock layer of external acoustic waves and a blowing-suction near the plate leading edge has been first demonstrated numerically. Essential suppression of density pulsations on the upper edge of boundary layer is distinctively visible though the oscillations of the SW did not change. Similar results were obtained in DNS performed by Fomin et al. [15] for external slow acoustic waves at $\mathrm{M}_{\infty}=21$. 


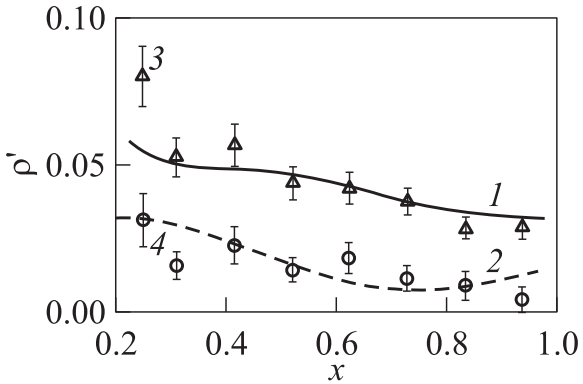

(a)

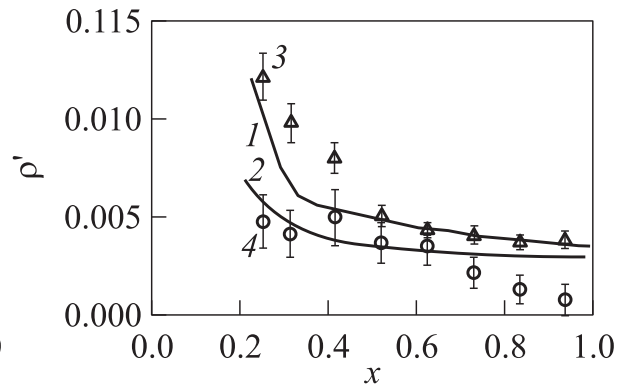

(b)

Figure 6 Comparison of experimental (symbols) and computed amplitudes of density fluctuations on the SW ( $a$ ) and on the boundary layer edge (b) for frequencies of $20 \mathrm{kHz}$ ( 1 and 3 , solid curves) and $40 \mathrm{kHz}$ (2 and 4, dashed curves) for perturbations of the blowing-suction type with $A=0.03$

The numerical data have been compared with results of measurements in the shock layer on a plate for zero angle of attack. The possibility of controlling the intensity of fluctuations in a hypersonic shock layer on a flat plate was also demonstrated in experiments for fast external acoustic waves. The experiments have been performed in the hypersonic nitrogen wind tunnel T-327A of ITAM at Mach number $\mathrm{M}=21$ and Reynolds number $\operatorname{Re}_{L}=1.44 \cdot 10^{5}$. The idea of this experiment is illustrated in Fig. 8.

Two types of disturbances were used in the experiment. First, controlled periodic disturbances of blowing-suction type were introduced into the shock layer by an oblique cylindrical gasdynamic whistle located under the plate in the vicinity of the leading edge. Second, fast acoustic waves of external, which were generated by a spark discharge in settling chamber of the nozzle and introduced by the whistle signal with a certain time delay $\Delta \tau$. This delay is responsible for the phase difference between these two types of disturbances.

Figure $9 b$ shows the measured amplitudes of density fluctuations in the cross section $x=0.63$ at the boundary-layer edge as functions of the delay of the spark signal at the whistle operation frequency. It is seen that a certain delay ensures almost complete suppression of density fluctuations at the boundarylayer edge. For comparison, computational data are shown by triangles, which are in good agreement with experimental data. The amplitudes are normalized to the amplitude of in-phase interaction of the sources of disturbances. The solid curve in Fig. $9 b$ approximates the experimental values in accordance with the law of interference of two sinusoidal waves of identical frequency and amplitude.

Figures $9 a$ and $9 d$ show the root-mean-square fluctuations of density in the cross section $x=0.5$ for two variants: with excitation of the shock layer by a 


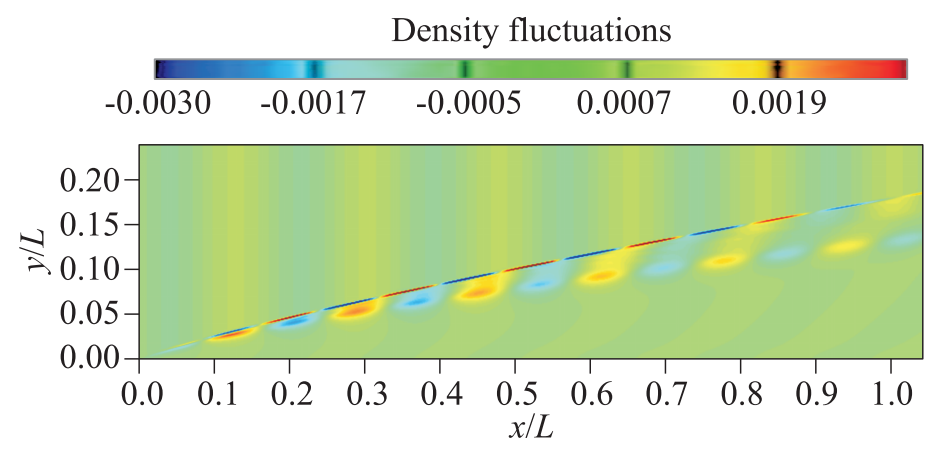

(a)

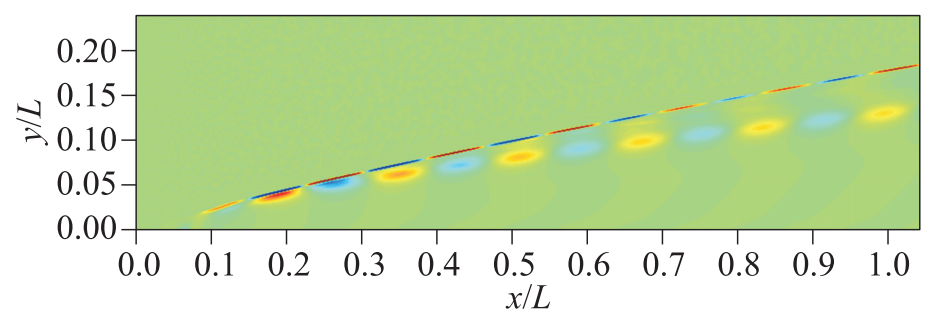

(b)

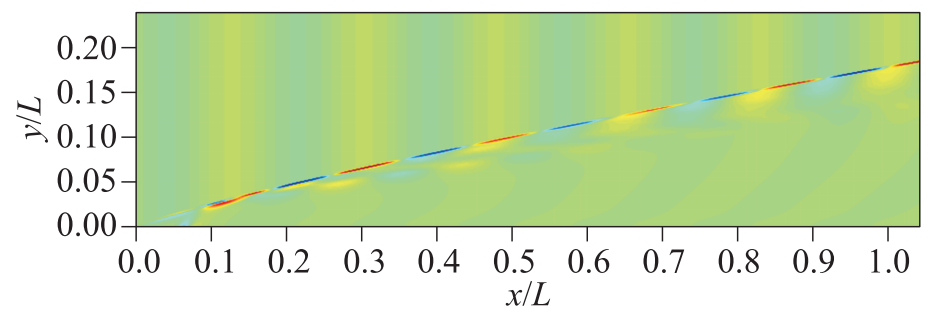

(c)

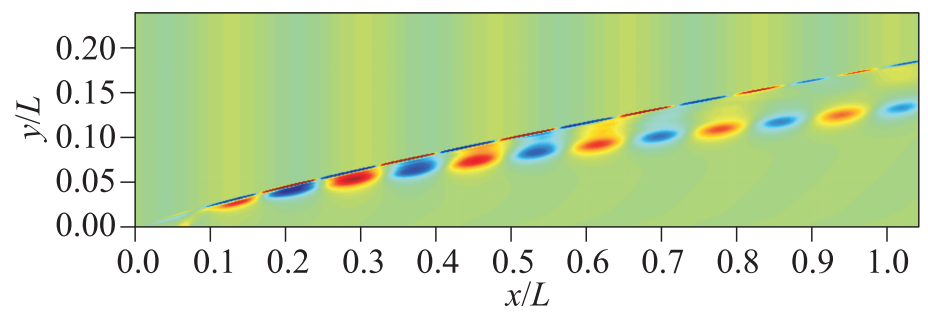

(d)

Figure 7 Fields of instantaneous density fluctuations in the shock layer for $f$ $=38.4 \mathrm{kHz}, \mathrm{M}_{\infty}=21, \operatorname{Re}_{L}=1.44 \cdot 10^{5}$ : (a) fast acoustic wave $A=0.0017$; (b) blowingsuction of the gas from the plate surface $A=0.0694$; $(c)$ antiphase action; and $(d)$ inphase action. (Refer Poplavskaya and Maslov, p. 213.) 


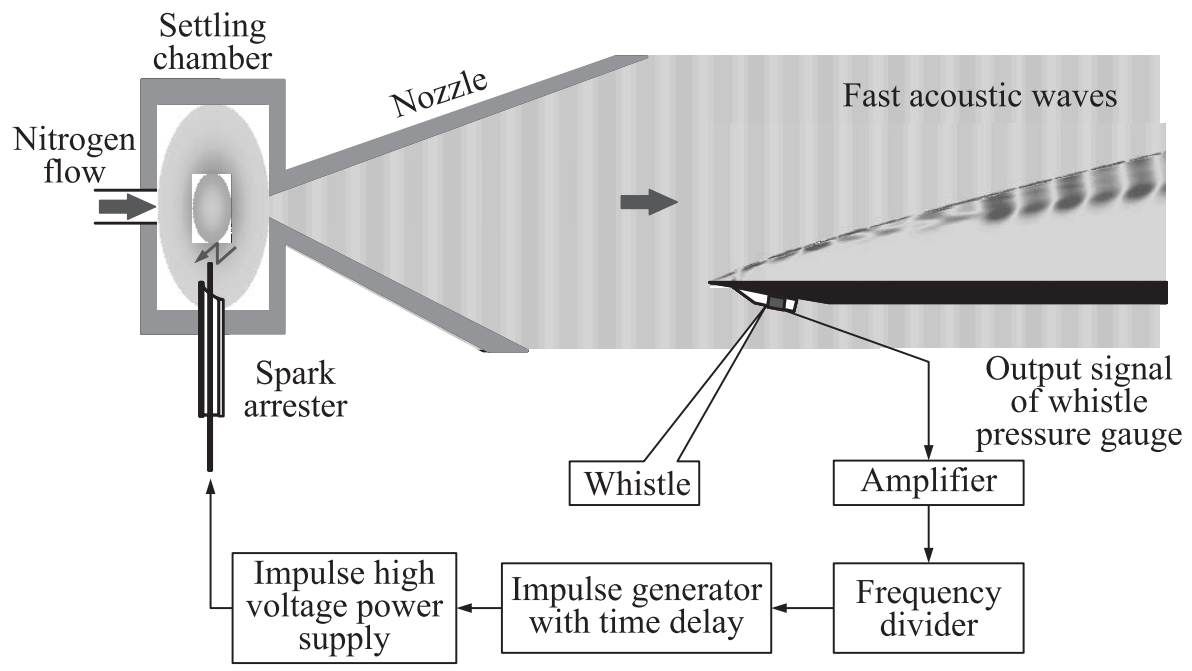

Figure 8 Arrangement of the experiment

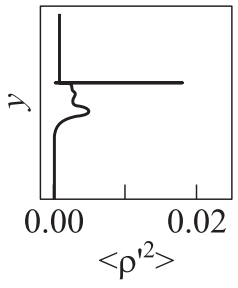

(a)

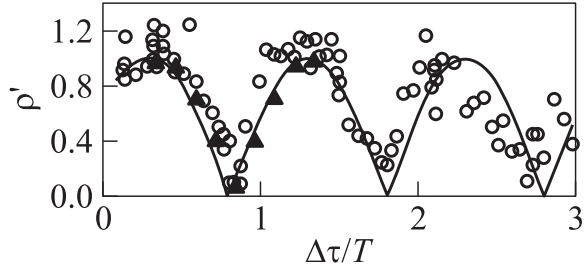

(b)

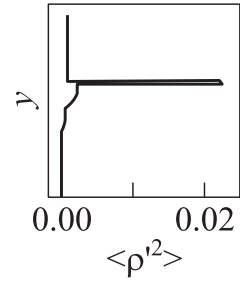

(c)

Figure 9 Numerically predicted and experimentally measured interference of two kinds of disturbances

fast external acoustic wave and with simultaneous action of an acoustic wave and antiphase blowing/suction. It is seen that the density fluctuations on the boundary-layer edge are almost completely suppressed.

\section{CONCLUDING REMARKS}

The present paper describes comprehensive numerical and experimental studies of receptivity and of disturbance evolution in a hypersonic VSL on a flat plate at a very high Mach number and moderate Reynolds numbers: 
- it is shown that the main specific feature of the mechanism of formation of the field of fluctuations in the case of shock-layer interaction with external acoustic disturbances and with perturbations of the blowing-suction type is generation and propagation of entropy-vortex perturbations inside the shock layer;

- it is shown that the field of fluctuations generated by external acoustic disturbances is qualitatively and quantitatively similar to the field of fluctuations generated by blowing/suction with the same frequency; and

- it is demonstrated that perturbations in the shock layer generated by external acoustic disturbances can be effectively suppressed by the action of blowing-suction-type perturbations.

\section{ACKNOWLEDGMENTS}

The authors are grateful to A. N. Kudryavtsev for the Navier-Stokes code, and also to S. G. Mironov and I. S. Tsyryulnikov for conducting the experiments and useful discussions.

This work was supported by the Russian Foundation for Basic Research (grant 09-08-00557) and by ADTP RNP 2.1.1/3963.

\section{REFERENCES}

1. Egorov, I. V., V. G. Sudakov, and A.V. Fedorov. 2006. Numerical simulation of receptivity of a supersonic boundary layer to acoustic disturbances. Fluid Dynamics 41(1):42-53.

2. Egorov, I. V., A. V. Fedorov, and V. G. Sudakov. 2008. Receptivity of a hypersonic boundary layer over a flat plate with a porous coating. J. Fluid Mech. 601:165-87.

3. Ma, Y., and X. Zhong. 2003. Receptivity of a supersonic boundary layers over a flat plate. Part 2. Receptivity to free-stream sound. J. Fluid. Mech. 488:79-121.

4. Ma, Y., and X. Zhong. 2005. Receptivity of a supersonic boundary layer over a flat plate. Part 3. Effects of different types of free-stream disturbances. J. Fluid Mech. 532:63-109.

5. Kudryavtsev, A. N., T. V. Poplavskaya, and D. V. Khotyanovsky. 2007. Application of high-order schemes in modeling unsteady supersonic flows. Math. Model. 19(7):39-55.

6. Suresh, A., and H. T. Huynh. 1997. Accurate monotonicity-preserving schemes with Runge-Kutta stepping. J. Comput. Phys. 136(Pt. 1):83-99.

7. Kudryavtsev, A. N., and D. V. Khotyanovsky. 2005. Numerical investigation of high speed free shear flow instability and Mach wave radiation. Int. J. Aeroacoustics $4(3-4): 267-86$. 
8. Shu, C.-W., and S. Osher. 1988. Efficient implementation of essentially nonoscillatory shock-capturing schemes, I. J. Comput. Phys. 77:439-71.

9. Kudryavtsev, A. N., S. G. Mironov, T. V. Poplavskaya, and I. S. Tsyryulnikov. 2006. Experimental study and direct numerical simulation of the evolution of disturbances in a viscous shock layer on a flat plate. J. Appl. Mech. Tech. Phys. 47(5):61727.

10. Kudryavtsev, A.N., A.A. Maslov, S. G. Mironov, T. V. Poplavskaya, and I. S. Tsyryulnikov. 2006. Direct numerical simulation of receptivity of a hypersonic boundary layer to natural and artificial disturbances. Vych. Tekhnol. 11(Pt. 1):10815. [In Russian.]

11. Maslov, A. A., T. V. Poplavskaya, and B. V. Smorodsky. 2004. Stability of a hypersonic shock layer on a flat plate. Comptes Rendus. Mech. 332(11):875-80.

12. Mironov, S. G., and A. A. Maslov. 2000. An experimental study of density waves in hypersonic shock layer on a flat plate. Phys. Fluids 12(6):1544-53.

13. Maslov, A. A., and S. G. Mironov. 1996. Experimental investigation of the hypersonic low-density flow past a half-closed cylindrical cavity. Fluid Dynamics 31:92832 .

14. McKenzie, J.F., and K. O. Westphal. 1968. Interaction of linear waves with oblique shock waves. Phys. Fluids 11:2350-62.

15. Fomin, V. M., A. N. Kudryavtsev, A. A. Maslov, S. G. Mironov, T. V. Poplavskaya, and I. S. Tsyryulnikov. 2007. Active control of disturbances in a hypersonic shock layer. Doklady Akademii Nauk 414(2):1-4. 